\title{
Electromagnetic Sources in a Moving Conducting Medium
}

\author{
Johannsen, Günther
}

Published in:

Journal of Mathematical Physics

Link to article, DOI:

$10.1063 / 1.1665740$

Publication date:

1971

Document Version

Publisher's PDF, also known as Version of record

Link back to DTU Orbit

Citation (APA):

Johannsen, G. (1971). Electromagnetic Sources in a Moving Conducting Medium. Journal of Mathematical Physics, 12(7), 1349-1351. https://doi.org/10.1063/1.1665740

\section{General rights}

Copyright and moral rights for the publications made accessible in the public portal are retained by the authors and/or other copyright owners and it is a condition of accessing publications that users recognise and abide by the legal requirements associated with these rights.

- Users may download and print one copy of any publication from the public portal for the purpose of private study or research.

- You may not further distribute the material or use it for any profit-making activity or commercial gain

- You may freely distribute the URL identifying the publication in the public portal

If you believe that this document breaches copyright please contact us providing details, and we will remove access to the work immediately and investigate your claim 


\title{
Electromagnetic Sources in a Moving Conducting Medium
}

\author{
GÜNTHER , JoHANNSEN \\ Danish Engineering Academy, Electrical Department, The Technical University of Denmark, Lyngby, Denmark
}

(Received 26 June 1970)

\begin{abstract}
The problem of an arbitrary source distribution in a uniformly moving, homogeneous, isotropic, nondispersive, conducting medium is solved. The technique used is to solve the problem in the rest system of the medium and then write the result in an appropriate four-dimensional, covariant form which is valid in any inertial system.
\end{abstract}

\section{INTRODUCTION}

Recently Besieris and Compton ${ }^{1}$ solved the problem of electromagnetic radiation by an arbitrary source in a uniformly moving, homogeneous, isotropic, nondispersive, conducting medium by making use of a relation between the fundamental solution of a radiation problem and that of a corresponding Cauchy initial value problem. An alternative method was provided by Chen and Yen, ${ }^{2}$ who applied judiciously chosen affine transformations to the pertinent differential equation.

It is the purpose of this paper to solve the same problem but in a different way. The most essential feature of the technique used in the present paper is that the problem is handled in the rest system $K^{\prime}$ of the medium "as long as possible," because the pertinent differential equations are much simpler in $K^{\prime}$. In fact the whole problem is solved in $K^{\prime}$ by making use of the known fundamental solution of the KleinGordon differential equation; the result is then transformed to an arbitrary inertial system $K$ by means of an appropriate tensor formulation.

We use Cartesian tensor notation as in Ref. 3. By a tensor we understand a tensor defined on the Lorentz transformation group. Latin subscripts run from 1 to 4 , Greek subscripts run from 1 to 3 . The coordinate $x_{4} \equiv i c t$, where $t$ is the time and $c$ the speed of light in vacuum; therefore, the metric tensor in 4-space is equal to the Kronecker symbol $\delta_{i j}$ (when Cartesian spatial coordinates are used) and we do not distinguish between contravariant and covariant tensors. Repeated subscripts obey the summation convention, and commas in subscripts denote partial differentiation with respect to coordinates (or covariant differentiation since the metric tensor is independent of the coordinates).

\section{THE POTENTIAL TENSOR (4-VECTOR)}

In any inertial system Maxwell's equations are

$$
\boldsymbol{\nabla} \times \mathbf{E}=-\frac{\partial \mathbf{B}}{\partial t},
$$

$$
\begin{aligned}
\boldsymbol{\nabla} \times \mathbf{H} & =\frac{\partial \mathbf{D}}{\partial t}+\mathbf{J}_{f}+\mathbf{J}, \\
\boldsymbol{\nabla} \cdot \mathbf{D} & =\rho_{f}+\rho, \\
\boldsymbol{\nabla} \cdot \mathbf{B} & =0
\end{aligned}
$$

where $\mathbf{E}, \mathbf{H}, \mathbf{D}, \mathbf{B}$ are familiar symbols for the field quantities, $\mathbf{J}_{f}, \rho_{f}$ denote the free current and charge densities, and $\mathbf{J}, \rho$ the externally applied current and charge densities.

In the rest system $K^{\prime}$ of the medium, the following constitutive relations are assumed to be valid:

$$
\begin{aligned}
\mathbf{D}^{\prime} & =\boldsymbol{\epsilon} \mathbf{E}^{\prime}, \\
\mathbf{B}^{\prime} & =\mu \mathbf{H}^{\prime}, \\
\mathbf{J}_{f}^{\prime} & =\sigma \mathbf{E}^{\prime},
\end{aligned}
$$

where $\epsilon, \mu, \sigma$ are the dielectric constant, the permeability and the conductivity, respectively.

It is well known ${ }^{4}$ that vector and scalar potentials $\mathbf{A}^{\prime}, \Phi^{\prime}$ can be introduced in $K^{\prime}$, satisfying the equations

$$
\begin{aligned}
& \nabla^{2} \mathbf{A}^{\prime}-\mu \epsilon \frac{\partial^{2} \mathbf{A}^{\prime}}{\partial t^{\prime 2}}-\sigma \mu \frac{\partial \mathbf{A}^{\prime}}{\partial t^{\prime}}=-\mu \mathbf{J}^{\prime}, \\
& \nabla^{2} \Phi^{\prime}-\mu \epsilon \frac{\partial^{2} \Phi^{\prime}}{\partial t^{\prime 2}}-\sigma \mu \frac{\partial \Phi^{\prime}}{\partial t^{\prime}}=-\frac{1}{\epsilon} \rho^{\prime},
\end{aligned}
$$

where we have assumed that $\rho_{f}^{\prime}=0$ because of the brevity of usual relaxation times.

$\mathbf{A}^{\prime}$ and $\Phi^{\prime}$ are connected by the gauge condition

$$
\boldsymbol{\nabla} \cdot \mathbf{A}^{\prime}+\mu \epsilon \frac{\partial \Phi^{\prime}}{\partial t^{\prime}}+\sigma \mu \Phi^{\prime}=0 .
$$

The translation of (3) into tensor language is given in Ref. 5 for the case $\sigma=0$. It is not difficult to show that if $\sigma \neq 0$, a single term has to be added so that the tensor wave equation for the potential tensor $A_{i}$ (consult Ref. 6) which is valid in any inertial system $K$ may be written as

$$
A_{i, n n}-\kappa A_{i, r s} U_{r} U_{s}-\sigma \mu A_{i, r} U_{r}=-S_{i},
$$

where $\kappa \equiv\left(n^{2}-1\right) / c^{2}, n \equiv c / c^{\prime}, c^{\prime} \equiv(\mu \epsilon)^{-\frac{1}{2}}, S_{i} \equiv$ $\mu\left(J_{i}+\kappa / n^{2} J_{r} U_{r} U_{i}\right), U_{r}$ is the velocity 4-vector, and 
finally $J_{i}$ is the current density 4-vector (consult Ref. 6) of the external source.

The tensor equation for the gauge condition turns out to be

$$
A_{r, r}-\kappa A_{r, s} U_{r} U_{s}-\sigma \mu A_{r} U_{r}=0 .
$$

\section{INTEGRATION OF THE TENSOR EQUATION FOR DAMPED WAVES}

The first-order term in (5) may be eliminated. Let $k_{i}$ denote a constant 4-vector (i.e., independent of the space-time coordinates $x_{r}$ ). Also, tensor functions $B_{i}$ and $T_{i}$ are defined by

$$
\begin{aligned}
B_{i} & \equiv A_{i} e^{-k_{r} x_{r}}, \\
T_{i} & \equiv S_{i} e^{-k_{r} x_{r}} .
\end{aligned}
$$

From (5) and (7) we derive

where

$$
B_{i, n n}-\kappa B_{i, r s} U_{r} U_{s}+l^{2} B_{i}=-T_{i},
$$

$$
l \equiv\left[k_{r} k_{r}-\kappa\left(k_{r} U_{r}\right)^{2}-\sigma \mu k_{r} U_{r}\right]^{\frac{1}{2}}
$$

and $k_{r}$ is subjected to the condition

$$
2 k_{i}-\left(2 \kappa k_{r} U_{r}+\sigma \mu\right) U_{i}=0 .
$$

Since $U_{i}^{\prime}=(0,0,0, i c),(10)$ is satisfied in $K^{\prime}$ if we define

$$
k_{i}^{\prime} \equiv\left(0,0,0, i \frac{\sigma \mu c}{2 n^{2}}\right)
$$

Because (10) is a tensor equation, it holds in any system of inertia $K$ since it holds in $K^{\prime}$. [In $K$ we can get $k_{r}$ from (11) by means of the tensor transformation law.]

$l$ is defined by (9) and transforms like an invariant under a Lorentz transformation. It is easily shown (in $K^{\prime}$ ) that

$$
l=\frac{1}{2} \sigma(\mu / \epsilon)^{\frac{1}{2}} .
$$

In $K^{\prime}(8)$ reduces to

$$
\left(\nabla^{\prime 2}+n^{2} \frac{\partial^{2}}{\partial x_{4}^{\prime 2}}+l^{2}\right) B_{i}^{\prime}=-T_{i}^{\prime}
$$

In preparation for the integration of this equation, consider

$$
\left(\nabla^{\prime 2}-(i n)^{2} \frac{\partial^{2}}{\partial x_{4}^{\prime 2}}-(i l)^{2}\right) G^{\prime}=-4 \pi \delta\left(u_{r}^{\prime}\right),
$$

where $u_{r}^{\prime} \equiv x_{r}^{\prime}-z_{r}^{\prime} ; z_{r}^{\prime}$ are parameters and $\delta\left(u_{r}^{\prime}\right) \equiv$ $\delta\left(u_{1}^{\prime}\right) \delta\left(u_{2}^{\prime}\right) \delta\left(u_{3}^{\prime}\right) \delta^{\star}\left(u_{4}^{\prime}\right) . \delta^{\star}\left(u_{4}^{\prime}\right)$ is a delta-function with purely imaginary argument, i.e., $\int_{-i \infty}^{i \infty} f(z) \delta^{\star}(z) d z=$ $f(0)$ for a great class of functions $f$.

Equation (13) is the Klein-Gordon equation for the time-dependent Green's function $G^{\prime}$. The solution of (13) for the whole space is given in Ref. 7 for real constants (in) and (il). It is readily seen that the solution also holds when (in) and (il) are purely imaginary; therefore

$G^{\prime}\left(x_{r}^{\prime}, z_{r}^{\prime}\right)$
$=\left\{\begin{array}{ll}\frac{\delta^{\star}\left(u_{4}^{\prime}-i n r^{\prime}\right)}{r^{\prime}}-i \frac{l}{n} \frac{J_{1}\left(l R^{\prime}\right)}{R^{\prime}} 1_{+}^{\star}\left(u_{4}^{\prime}-i n r^{\prime}\right), & \frac{u_{4}^{\prime}}{i}>0 \\ 0, & \frac{u_{4}^{\prime}}{i}<0\end{array}\right.$,

where

$$
\begin{aligned}
R^{\prime} & \equiv\left[r^{\prime 2}+\left(u_{4}^{\prime} / n\right)^{2}\right]^{\frac{1}{2}}, \\
r^{\prime} & \equiv\left(u_{v}^{\prime} u_{v}^{\prime}\right)^{\frac{1}{2}} .
\end{aligned}
$$

$J_{1}$ is the Bessel function of first kind and first order, and $1_{+}^{\star}$ denotes the unit step function with purely imaginary argument, i.e.,

$$
1_{+}^{\star}(x)=\left\{\begin{array}{l}
1, x / i \geq 0 \\
0, x / i<0
\end{array}\right.
$$

By means of $G^{\prime}$ we are able to write down an integral representation for the potentials $A_{i}^{\prime}$ connected with $B_{i}^{\prime}$ by (7):

$$
\begin{aligned}
A_{i}^{\prime}\left(x_{r}^{\prime}\right)=\frac{1}{4 \pi} \iiint_{-\infty}^{\infty} \int_{-i \infty}^{i \infty} e^{k_{r}^{\prime} u_{r}^{\prime}} G^{\prime}\left(x_{r}^{\prime}, z_{r}^{\prime}\right) \\
\times S_{i}^{\prime}\left(z_{r}^{\prime}\right) d u_{1}^{\prime} d u_{2}^{\prime} d u_{3}^{\prime} d u_{4}^{\prime} .
\end{aligned}
$$

\section{TRANSFORMATION TO AN ARBITRARY INERTIAL SYSTEM}

Let $a_{j i}$ be the transformation matrix for a proper Lorentz transformation, i.e., $x_{r}=a_{r s} x_{s}^{\prime}$. Multiplying (16) by $a_{j i}$, we see that the left side is equal to $A_{j}\left(x_{r}\right)$ because $A_{j}$ is a tensor. $a_{j i}$ may be taken under the integral and, since $S_{i}$ is a tensor, $a_{j i} S_{i}^{\prime}\left(z_{r}^{\prime}\right)=$ $S_{j}\left(z_{r}\right)$ if the Lorentz transformation is also applied to the integration variables, i.e., if $z_{r}=a_{r s} z_{s}^{\prime}$ which implies $u_{r}=a_{r s} u_{s}^{\prime}$. Furthermore, $k_{r}$ is a tensor so that $e^{k_{r^{\prime}} u_{r}^{\prime}}=e^{k_{r} u_{r}}$.

Next we investigate how the Green's function $G^{\prime}$ is transformed. Without loss of generality, we choose $a_{r s}$ so that $x_{1}=x_{1}^{\prime}, \quad x_{2}=x_{2}^{\prime}, \quad x_{3}=\gamma\left(x_{3}^{\prime}+i \beta x_{4}^{\prime}\right)$, $x_{4}=\gamma\left(x_{4}^{\prime}-i \beta x_{3}^{\prime}\right)$, where $\gamma \equiv\left(1-\beta^{2}\right)^{-\frac{1}{2}}, \beta \equiv v / c$, and $v$ is the velocity of $K$ relative to $K^{\prime}$.

Consider a three-dimensional hypersurface in Minkowski space, which in $K^{\prime}$ is given by $R^{\prime 2}=0$, $u_{4}^{\prime} / i>0$ (Fig. 1) (cf. Ref. 3). In order to express the surface independently of the inertial system, we define

$$
R \equiv\left[u_{r} u_{r}+\kappa\left(\frac{u_{r} U_{r}}{n}\right)^{2}\right]^{\frac{1}{2}} .
$$

Obviously $R$ is an invariant function of $u_{r}$, and it is easily seen that $R=R^{\prime}$ in $K^{\prime}$. Therefore, the 


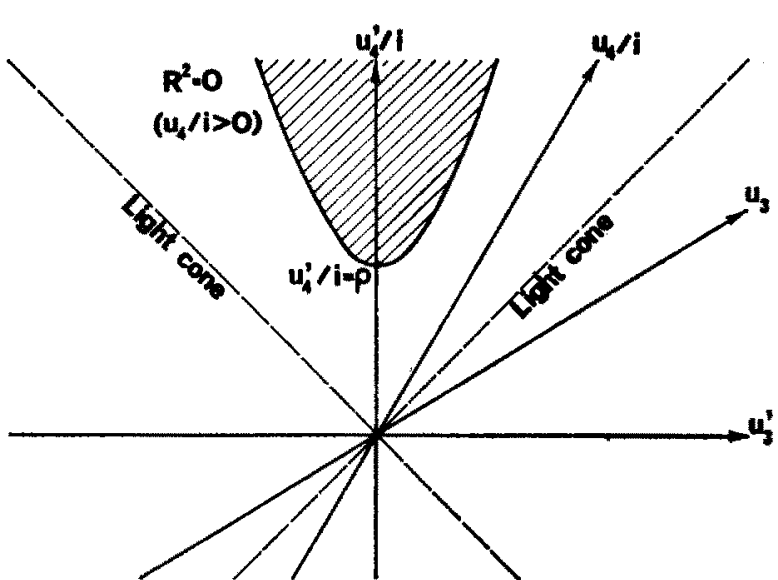

Frg. 1. Location of zeros of the function $R^{2}$ in Minkowski 4-space for $u_{4} i i>0$.

hypersurface is also given by $R^{2}=0, u_{4} / i>0$ because it is located entirely in that part of Minkowski space where the condition $u_{4} / i=\left(x_{4}-z_{4}\right) / i>0$ is valid in any inertial system $K$.

$u_{4}$ is purely imaginary so $R^{2}$ may be negative, which is the case inside the hatched domain in Fig. 1. The roots of $R^{2}=0$ are given by (cf. Ref. 3 )

$$
\begin{aligned}
\frac{u_{4}}{i} & =\tau_{ \pm} \\
& \equiv \frac{n \beta}{1-(n \beta)^{2}}\left[\left(n-\frac{1}{n}\right) u_{3} \pm\left(\frac{1}{\beta}-\beta\right)\left(u_{3}^{2}+a \rho^{2}\right)^{\frac{1}{2}}\right],
\end{aligned}
$$

where $a \equiv\left[1-(n \beta)^{2}\right] /\left(1-\beta^{2}\right), \rho \equiv\left(u_{1}^{2}+u_{2}^{2}\right)^{\frac{1}{2}}$.

It is seen that $n \beta<1$ implies $\tau_{+}>0, \tau_{-}<0$. In the domain $u_{4} / i>0$ the equation $R^{2}=0$ defines a one to one correspondence between $u_{3}$ and $u_{4}$ (for given $\rho$ ). This is not the case for $n \beta>1$ (Cerenkov region) because $\tau_{\mp}>0$ for $u_{4} / i<-\rho|a|^{\frac{1}{2}}$, and both roots are complex or do not belong to the domain $u_{4} / i>0$ for $u_{4} / i>-\rho|a|^{\frac{1}{2}}$.

From the preceding remarks we conclude that the step function in (14) may be written in covariant form as $1_{+}\left(-R^{2}\right), u_{4} / i>0$.

As to the $\delta$ function in (14), we have

$$
\begin{array}{r}
\frac{\delta^{\star}\left(u_{4}^{\prime}-i n r^{\prime}\right)}{-\left(d R^{2} / d u_{4}^{\prime}\right)_{u_{4}^{\prime}=i n r^{\prime}}}=\frac{\delta^{\star}\left(u_{4}^{\prime}-i n r^{\prime}\right)}{i(2 / n) r^{\prime}}=\delta\left(-R^{2}\right), \\
u_{4} / i>0 .
\end{array}
$$

Finally we observe that the limits of integration in (16) remain unchanged because a Lorentz transformation is a one-to-one mapping of the Minkowski space on itself.

We are now able to write down the covariant forms of (14) and (16) valid in an arbitrary inertial system:

$$
\begin{aligned}
& A_{i}\left(x_{r}\right)=\frac{1}{4 \pi} \iiint_{-\infty}^{\infty} \int_{-i \infty}^{i \infty} e^{k_{r} u_{r}} G\left(x_{r}, z_{r}\right) \\
& \times S_{i}\left(z_{r}\right) d u_{1} d u_{2} d u_{3} d u_{4}, \\
& G\left(x_{r}, z_{r}\right) \\
& =\left\{\begin{array}{ll}
i \frac{2}{n} \delta\left(-R^{2}\right)-i \frac{l}{n} \frac{J_{1}(l R)}{R} 1_{+}\left(-R^{2}\right), & \frac{u_{4}}{i}>0 \\
0, & \frac{u_{4}}{i}<0
\end{array} .\right.
\end{aligned}
$$

When $n \beta<1$,

$$
\begin{aligned}
& i \frac{2}{n} \delta\left(-R^{2}\right)=i \frac{2}{n} \frac{\delta^{\star}\left(u_{4}-i \tau_{+}\right)}{n-\left(d R^{2} / d u_{4}\right)_{u+i \tau_{+}}}=\frac{\delta^{\star}\left(u_{4}-i \tau_{+}\right)}{\left(u_{3}^{2}+a \rho^{2}\right)^{\frac{1}{2}}}, \\
& u_{4} / i>0 \text {. }
\end{aligned}
$$

When $n \beta>1$, both roots $\tau_{ \pm}$play a part as pointed out before. In this case it turns out that

$$
\begin{gathered}
i \frac{2}{n} \delta\left(-R^{2}\right)=\frac{\delta^{\star}\left(u_{4}-i \tau_{+}\right)+\delta^{\star}\left(u_{4}-i \tau_{-}\right)}{\left(u_{3}^{2}+a \rho^{2}\right)^{\frac{1}{2}}}, \\
u_{4} / i>0 .
\end{gathered}
$$

If the medium is nonconductive, i.e., $\sigma=0$, then $k_{r} u_{r}=0$ and $l=0$ [cf. (11) and (12)], furthermore, the second term in (21) evidently vanishes. This problem has been investigated previously by Compton, ${ }^{8}$ Lee and Papas, ${ }^{9}$ Tai, ${ }^{10,11}$ and the author. ${ }^{3}$ As pointed out by Tai, ${ }^{11}$ the first term in (21) is equivalent to the corresponding expression found by Compton. ${ }^{8}$

As to the general case $(\sigma \neq 0)$, the result given by Besieris and Compton ${ }^{1,12}$ is in error ${ }^{13}$ due to miscalculation, and there is a formal error in Ref. $2,{ }^{14}$ so the author hopes deeply that he is right in asserting that the results in Refs. 1 and 2 can be brought into agreement with the results given here.

${ }^{1}$ I. M. Besieris and R. T. Compton, Jr., J. Math. Phys. 8, 2445 (1967).

${ }^{2}$ K. C. Chen and J. L. Yen, J. Math. Phys. 9, 2081 (1968).

${ }^{3}$ G. Johannsen, J, Math. Phys. 11, 3251 (1970).

* D. S. Jones, The Theory of Electromagnetism, Vol. 47 (Pergamon, New York, 1964).

${ }^{5}$ J. M. Jauch and K. M. Watson, Phys. Rev. 74, 950 (1948).

- C. Maller, The Theory of Relativity (Oxford U.P., London, 1952).

${ }^{7}$ P. M. Morse and H. Feshbach, Methods of Theoretical Physics, Vol. I (McGraw-Hill, New York, 1953), pp. 854-857.

${ }^{8}$ R. T. Compton, Jr., J. Math. Phys. 7, 2145 (1966).

${ }^{9}$ K. S. H. Lee and C. H. Papas, J. Math. Phys. 5, 1688 (1964). (1965)

11 C. T. Tai, J. Math. Phys. 8, 646 (1967).

12 I. M. Besieris, J. Math. Phys. 8, 409 (1967).

${ }_{13}$ As pointed out in Ref. 2, there is an algebraic error in Eq. (18a), Ref. 1 ( $v$ should be replaced by $\gamma v)$; furthermore, the factor before the second term of (59), Ref. 12, is not correct, which in turn influences the results in Ref. 1 .

${ }^{14}$ In Eq. (21) etc., Ref. 2, the argument of the Bessel function is in error. 